\title{
Transitions in Mathematics Education: The Panel Debate
}

\author{
Ghislaine Gueudet, Marianna Bosch, Andrea A. diSessa, \\ Oh Nam Kwon and Lieven Verschaffel
}

\begin{abstract}
The Transitions in Mathematics Education panel during the ICME-13 conference consisted of two parts. In the first part, the panelists presented particular questions addressed and answered them according to their various perspectives (some of them cognitive, others more sociocultural). This first part was published as a survey before the conference (Gueudet et al. in Funds of knowledge: Theorizing practices in households, communities, and classrooms. Erlbaum: Mahwah, NJ, 2016). In the present text, we briefly review this first part but mainly focus on the second part of the panel. In the second part, the panelists answered questions about the survey concerning the arithmetic-algebra transition, the possible use of boundary objects to build links and bridges, the role of technical work in the continuity/discontinuity of the learning process, and the possible contributions of students in helping to ease transitions. These answers are developed and presented here.
\end{abstract}

G. Gueudet $(\bowtie)$

CREAD, ESPE Bretagne UBO, 153 Rue Saint Malo, 35000 Rennes, France

e-mail: ghislaine.gueudet@espe-bretagne.fr

M. Bosch

IQS School of Management, Universitat Ramon Llull, Via Augusta, 390,

08022 Barcelona, Spain

e-mail: marianna.bosch@iqs.url.edu

A.A. diSessa

Graduate School of Education, University of California, Berkeley, CA 94720, USA

e-mail: disessa@berkeley.edu

O.N. Kwon

Seoul National University, Gwanak-Gu Gwanak-Ro 1, 151-748 Seoul, Korea

e-mail: onkwon@snu.ac.kr

L. Verschaffel

Center for Instructional Psychology and Technology, Education and Training Research

Group, Katholieke Universiteit Leuven, Van Den Heuvel Instituut (Room Nr. 05.71),

Dekenstraat 2, 3773, B-3000 Leuven, Belgium

e-mail: Lieven.Verschaffel@ppw.kuleuven.be

(C) The Author(s) 2017

G. Kaiser (ed.), Proceedings of the 13th International Congress on Mathematical

Education, ICME-13 Monographs, DOI 10.1007/978-3-319-62597-3_7 
Keywords Boundary object - Curriculum - Discontinuity - Institutions • Transition

This text follows the content and organization of the ICME-13 panel about transitions in mathematics education. The panel started with individual presentations, putting forward different perspectives on transitions. After this first part, some questions, mainly raised by participants of the online panel preparation forum, were discussed from these different perspectives. We start here by briefly reviewing the content of the individual presentations (Section "Different Views on Transitions, a Survey") and then develop answers to the questions (Section "Addressing Transition Questions with Different Perspectives"), showing how contrasting views can complement each other in mathematics education research.

\section{Different Views on Transitions, a Survey}

The ICME-13 panel about "transitions in mathematics education" was grounded in a literature survey on this topic (Gueudet Bosch, diSessa, Kwon, \& Verschaffel, 2016). We recommend reading the complete survey, which is available online at no cost. However, in this section a short version of the survey is developed that is sufficient to understand the various perspectives presented in the panel.

\section{Which Transitions?}

Many different kinds of transitions have been studied in the research literature. In our survey, we mainly addressed two kinds of change: (a) conceptual change and learning as a transition process and (b) transitions as people move between social groups or contexts with different mathematical practices.

Some researchers have studied changes within the mathematical content from an epistemological perspective, sometimes drawing on the history of mathematics (e.g., Dorier, 2000). Research from this perspective has also studied transitions during students' learning; these cognitive transitions can be viewed within various theoretical frameworks and concern specific mathematical topics but also more general issues, such as the transition between different thinking modes (e.g., Tall, 2002).

Other authors consider mathematics to be shaped by groups of people who develop shared mathematical practices; they investigate transitions between different such groups using a sociocultural perspective (Crafter \& Maunder, 2012). Relevant groups can be of different natures; for example, they can correspond to different languages, different mathematical practices, and a kind of "permanent transition” (Ríordáin \& O’Donoghue, 2011). Groups involved in a transition can 
also be moving between different teaching institutions (such as between primary and secondary school) or from a teaching institution and the workplace. These two last cases (two teaching institutions or a teaching institution and a workplace) were studied in specific sections of the panel, as described below; they both correspond to "local" transition, happening at a given moment in time. The corresponding studies often identify discontinuities and sometimes design teaching experiments in order to smooth the relevant transition.

\section{Continuity Versus Discontinuity in Learning Difficult Concepts}

This presentation focused on the cognitive processes by which prior (before instruction) concepts are transformed into normative understanding. This complements - and does not replace-sociocultural perspectives, which focus more on culture and membership, and less on concepts per se. In particular, we trace the history and current status of a contest between "revolutionary" theories of conceptual change, and "evolutionary" ones, which emphasize continuities over categorical discontinuities.

The early history of this contest in both mathematics and science education greatly favored the discontinuous point of view. In mathematics, the idea of "epistemological obstacles" was imported from philosophical work and, broadly speaking, it characterized prior stages of thinking as involving ideas that are persistent, unavoidable, and relatively monolithic in that they required substantial "ruptures" or discontinuities in thinking to overcome. In science education also, the philosophical literature was influential. Thomas Kuhn's ideas of incommensurability between paradigms were imported and became deeply ingrained in educational studies of conceptual change.

Without presuming that the relevant issues are settled, the presentation surveyed the advance of more continuist approaches to conceptual change over the years, using recent studies to illustrate orientations, relevant methodologies, and results in both mathematics and mathematically oriented physics. In general, the presentation advocated methodologies that pursue a finer grain size of analysis (with respect to conceptual detail and with respect to smaller time scales) than has been typical in the past. A smaller grain size makes it possible to see prior results and orientations as perhaps unnecessarily dichotomous and is amenable to more nuanced and complex descriptions, parallel to the trend noted concerning the relation of in- and out-of-school ways of thinking in the presentation of Lieven Verschaffel (Section "Transitions Between in- and Out-of-School Mathematics").

The presentation concluded by describing major differences between continuous and dichotomous views of conceptual change from the perspective of teachers and other educational professionals. 


\section{Double Discontinuity Between Secondary School \\ Mathematics and University Mathematics: Focusing on Mathematical Knowledge for Teaching}

This presentation dealt with two transitions that prospective teachers experience in becoming professionals-Klein's double discontinuity: from secondary school to university and then from university to teaching in secondary school. It provided an overview of the current state of the art in the context of teacher education in order to provide a deeper understanding of the double discontinuity phenomenon with a special focus on mathematical knowledge.

The distinction between subject matter knowledge and pedagogical content knowledge for teacher education has proved practically useful and has been employed in numerous studies. However, the assessment of teachers' subject matter knowledge and pedagogical content knowledge requires a theory of the subject in question and of its knowledge. There is broad consensus that these two components of professional knowledge cannot simply be equated.

There are two principal approaches to interconnecting these different kinds of mathematical knowledge that may be used together profitably: one that adds aspects of the new university discourse slowly and step by step and one that develops university-level problems starting with school mathematics. A third approach is to develop courses that explicitly integrate subject matter knowledge with pedagogical content knowledge in mathematics and the didactics of mathematics.

Klein's notion of a double discontinuity between university mathematics and school mathematics has proved to be extremely fruitful and can be seen to constitute the core of mathematics teacher education in both theoretical and practical respects.

\section{Transitions Between Teaching Institutions}

During their studies, students experience many transitions between educational institutions: from preschool to primary school, from primary to secondary school, sometimes from lower to higher secondary school or technical college, and from higher secondary to university. These transitions mean changes in many senses and are often seen as an important source of difficulties - rarely as opportunities - for the development of the students' learning. While research on transitions has mainly focused on the passage from secondary to tertiary education, some studies are starting to use a similar perspective to consider the passage from primary to lower secondary level.

We propose using different levels of specificity when considering the main research findings in the study of these two types of transitions, from the more general ones related to the culture and organizational rules of the educational institution to the more concrete ones linked to the ways of dealing with the various 
components of the mathematical content. Surprisingly, some of the phenomena pointed out as difficulties in the passage from primary to secondary level seem to reappear in the passage from secondary school to university. However, the treatment of these difficulties appears to be clearly asymmetric. Looking forward to ameliorating difficulties of transitions, in the first case the "receiver" institution (secondary education) seems to require more change to become closer to the "sender" institution. In the second case it is again the secondary level, here acting as "sender," which is questioned, while the university's prevailing pedagogical and mathematical organization remains almost unquestioned. Therefore, it seems important to take into account that transitions happen between institutions maintaining a certain hierarchical relationship in their raison d'être as preparatory schools as well as in their distance from scholarly mathematics, secondary education assuming an ambiguous role between the education for all and the preparation for tertiary studies.

\section{Transitions Between in- and Out-of-School Mathematics}

Besides the processes of conceptual change and the transitions from one instructional level or section to another described in the other sections of this review, research in mathematics education has also been confronted with the multifold transitions between in- and out-of-school mathematics. Within this topic, we distinguish between (1) the transition from prior-to-school to school mathematics and (2) the transitions from out-of-school to school mathematics (and vice versa). While the first kind of transition may be considered a non-reversible process, the second kind may be construed as an interaction - a "permanent transition" between two contexts.

The transition from prior-to-school to school mathematics is currently dominated by two quite different lines of research. First, there has been the very productive and influential line of neuro-cognitive research on children's early number sense, its development, and its relation to school mathematics. In a complementary line, researchers have approached the transition from prior-to-school to school mathematics from a sociocultural perspective, wherein it is primarily conceived as a set of processes whereby individuals "cross borders" from one cultural or educational context or community to another.

The research literature on the transitions from out-of-school to school mathematics (and vice versa) has been dominated by three main themes: (1) exploration of out-of-school mathematical practices and cultures (in comparison to mathematics learned at school), (2) difficulties in the transition between out-of-school and school mathematics, and (3) attempts to facilitate and exploit these transitions. It is interesting to note that the older extremely dichotomous descriptions of the pitfalls and merits of in- and out-of-school (learning) practices have been replaced by more nuanced and complicated analyses of these different kinds of mathematical 
practices and of the various types of transitions between them and the affordances of these transitions for educational purposes.

\section{Addressing Transition Questions with Different Perspectives}

The second part of panel discussed four questions, presented below, engaging the various perspectives evoked above. The question proposed to the panelists was formulated as:

"With the perspective you presented, can you say something (and what) about the following theme or question:"'(the corresponding list of themes are given below as the titles of the subsections).

The panelists' answers are presented as a discussion (MB for Marianna Bosch, AdS for Andrea diSessa, ONK for Oh Nam Kwon, and LV for Lieven Verschaffel). For each question, two or three of the panelists responded.

\section{The Transition from Arithmetic to Algebra}

AdS: The transition from arithmetic to algebra should be an excellent example of the same considerations that I elaborated in my primary essay. Typical of the history described in my essay, the literature so far seems biased toward discontinuity. Researchers expect that some compact description of the essential difference between arithmetical and algebraic reasoning will tell the story. In contrast, I believe that if we look closely (finer conceptual and temporal grain sizes), we will find definitively incremental learning paths.

One problem with prior research is that different dimensions of change have not been adequately disentangled. Change appears too difficult, until we can "divide and conquer." Learning requires consideration of multiple threads, and each thread is, I maintain, less dichotomous appearing. For example, in much student work in the literature, I see learning difficulties that are associated with a general expertise with the nature of representations. These are almost never separated from the larger picture. A simple example is that students can't, or even will refuse to, answer problems given in a very slightly unusual representational system, say, with the $x$ axis vertical and $y$-axis horizontal. The ironic fact is that, based on some of our work with sixth-grade children (diSessa, 2004), teaching about representations seems surprisingly easy. It's just that school simply does not engage this learning thread.

A second thread that is unengaged in current instruction is the very nature of mathematics: the child's view of the mathematical enterprise. When the world shifts from numbers and procedures to relations and processes on relations, we should 
most certainly engage our students in thinking what that entails and feels like. I barely see any such recognition in contemporary curricula, and we may be suffering for the lack of attention.

Continuist views are still relatively new in mathematics, even more so concerning the arithmetic-to-algebra transition. However, work is progressing. For example, Mariana Levin (2012) has taken a microgenetic look at learning in pre-algebra. In her case study of a student developing the idea of linear extrapolation, she shows (1) the deep interaction between conceptual and procedural knowledge and (2) a lot of the typical phenomena of the continuist perspectives: many small learning events and a high degree of contextualization, which requires more time but no big jumps.

MB: The passage from arithmetic to algebra is one of the most often considered when addressing the question of transitions or discontinuities in school mathematics. If we look at school mathematics as a growing process of mathematization, this specific passage attracts the attention of teachers, educators, and researchers much more than others, such as the passage from Euclidean to analytic geometry, from algebra to functions, or from the consideration of elementary deterministic processes to stochastic ones. What is the specificity of this transition?

From an institutional perspective, a reason can be found in the specific role played by algebra in the structuring of old mathematical curricula. In many countries and for many decades, at least until the global reform of New Math during the 1960s and 1970s, school curricula were organized in three domains: arithmetic, algebra, and geometry. Students went to primary school to learn arithmetic, together with some practical applications and basic elements of measure and geometry. This represented the common mathematical culture of the broad (educated) population. Those who went to secondary school (relatively few, in some countries) had to learn algebra and analytical geometry. In any case, algebra marked the entrance to post-compulsory secondary education.

The difficulties attributed to the transition between arithmetic and algebra in current school processes maintain a semblance of the selective role formerly played by algebra. Today, algebra is still considered to be the first "abstract" content students should learn and may also be the first time students have difficulty in "giving meaning" to the mathematical practices they are asked to do. As educational researchers, we have to protect ourselves from this cultural perspective on school mathematical content and consider all of them from a unique and "uncontaminated" point of view (Bosch, 2015). In this context, the arithmetic-algebra transition should be approached by questioning the construction of the entire curriculum, that is, the whole process of mathematization as it is introduced to students. This questioning has to reach the traditional sequence of school mathematical content. Otherwise, we could be falling into the misconception, denounced by Paulos (2001), of considering mathematics "as a completely hierarchical subject. First comes arithmetic, then algebra, then calculus, then differential equations, abstract algebra, complex analysis, and so on. This is not necessarily so" (p. xiii). 
Another important question that the arithmetic-algebra transition indirectly points out is the lack of a coherent and explicit discourse about the school mathematical curriculum "for all" and the role of algebra in this curriculum. The "algebra controversy" began years ago in the United States. Hacker's (2012) article, "Is algebra necessary?" explains it very well. When a society doubts the importance and utility of the mathematical content all citizens should learn, instruction of this knowledge is half-hearted at best.

\section{What Are Appropriate or Promising “Boundary Objects” that Can Play a Contributing Role in Helping Students to Make the Transition?}

$\mathbf{L V}$ : The first example that I think of is "word problems." These tasks were in existence already thousands of years ago to help pupils, from a young age on, (1) to see the links between mathematics lessons learned at school and the out-of-school world wherein to-be-learned mathematics has to be applied and (2) to establish productive transitions between the world of in- and out-of-school mathematics. This is what is typically called the "application function of word problems" (Verschaffel, Greer, \& De Corte, 2000). So, in a way, word problems are intrinsically at the boundary of these two different worlds.

Meanwhile, we all know from a lot of theoretical analyses and empirical work that word problems do not play their application function very well. Indeed, word problems have gradually evolved into another type of school mathematics task that has little to do with authentic and complex mathematical modeling and application situations in the real world outside school. Accordingly, the actions that pupils perform when confronted by these word problems have little in common with what we would call genuine mathematical modeling and applied problem solving. As such, word problems can hardly be considered appropriate or promising "boundary objects" that help pupils make the transition between in- and out-of-school mathematics.

In reaction to this evaluation, mathematics educators have made numerous and varied attempts to make word problems more authentic so that they are better "simulations" of mathematical modeling and application problems situated in the real world. Some have gone even further and replaced these (mainly) verbal problems by rich, authentic, complex problem-solving contexts offering ample opportunities for genuine mathematical modeling and applied problem solving (with help of video and computer technology; Cognition and Technology Group at Vanderbilt, 1997).

However, as several authors have argued (e.g., Gravemeijer, 1997; Verschaffel et al., 2000), it is not always possible, and probably even not always necessary, to include the complexity of the out-of-school reality in the mathematics classroom. If you always try to accommodate all reality in your mathematics class, things may get 
out of hand. You open a "Pandora's box" (Verschaffel et al., 2000). Moreover, traditional word problems may still have their function in elementary mathematics education (alongside more complex, authentic, and challenging genuine modeling and application problems), particularly as a convenient means to create strong links between the basic mathematical operations and prototypically "clean" model situations (with little room for endless discussions about the situational complexities that might jeopardize this link). However, a lot can be accomplished by talking about the issues. For instance, during whole-class discussions, upper elementary school pupils can learn to differentiate between standard word problems (S-items) and problems that are problematic from a realistic modeling point of view (P-items), such as the rope item that I used in my talk ("A man wants to have a rope long enough to stretch between two poles $12 \mathrm{~m}$ apart, but he has only pieces of rope $1.5 \mathrm{~m}$ long. How many of these pieces of rope would he need to tie together to stretch between the poles"). Or, to give another example, take the following calendar joke "Ten birds are sitting in a tree. A hunter comes and shoots three of them. How many birds are still sitting in that tree afterwards?" Discussing with pupils these P-items or jokes - which I would consider to be "boundary objects" par excellence - are, in my view, excellent and important activities to understand the roles of simplification and consequent approximation in mathematical modeling and applied problem solving.

MB: In the case of the transition between secondary and tertiary education, an interesting boundary object can be the so-called bridging courses organized in different universities to smooth the gap between upper-secondary school and university. They are a good example of the ambiguity between individual interventions and institutional practices. We can construe their main goal as helping fist-year students smooth difficulties with the new learning processes they encounter at the university. Therefore, students are offered various courses, depending on the country and university - now most of them are online - where mainly secondary school contents are revisited and a few more advanced notions are introduced. These courses are usually taught in a short period, although they can also last a whole academic year, and are rarely recommended to all students, usually only for those who feel or are considered to be less prepared.

However, in their aim to help individuals smooth the transition, the effect produced at an institutional level is to reinforce the frontier between secondary and tertiary education. In a sense, bridging courses are the message transmitted by university to secondary education about what mathematical skills, competencies, practices, and contents students need but do not have. The subliminal message then is: "The students' preparation is not good enough and we are compelled to do the work you have not done." At the same time, the bridging courses tend to highlight the differences between both institutions instead of stressing the commonalities, which could offer students a link they might not be able to see. For instance, some studies (Serrano, Bosch, \& Gascón, 2007; Sierpinska, 2006) show how the bridging courses can have a reverse effect and contribute to increasing the gap between institutions. Instead of facilitating the entrance to the new culture and its practices, the courses propose intensive work based on "filling the gaps" in the required basic 
knowledge, thus reinforcing and rigidifying the old relationships to the old mathematical contents. As indicated by Fonseca, Bosch and Gascón (2004), university mathematical content could explicitly emerge from questions and limitations of secondary school mathematics, proposing a way to develop the old contents towards more complete, intertwined, and powerful constructions. However, this ambitious process requires global changes in university mathematics education that cannot be accomplished in summer courses. Up to now, the bridging courses appear as a coup de force of the tertiary institution to clearly establish entrance requirements for the new students without any attempt to adapt its own practices to the newcomers or to the feeding institutions. The strategy in this way is very different from procedures aimed at smoothing the passage between primary and secondary education. In the latter, teachers from both institutions meet to exchange practices and increase shared activities, assuming the principle that changes are necessary on both sides of the transition.

\section{What About Learning Technical, Procedural Work in the Acquisition of Concepts? How Does It Contribute to the Continuity/Discontinuity of the Learning Process?}

AdS: While not a scientific result, the following shocking experience profoundly influenced my research program. Long ago, I engaged in an interviewing study involving dozens of MIT freshman physics students across several years. In the first interview, I always asked about students' experience with physics in high school. These MIT students were excellent and well prepared and they all said they did well and got an A. However, almost all of them added, "But I didn't understand anything." I believe that comment was insightful and showed a strong aesthetic about understanding and good judgment about it. Most of the students did not really understand a lot of physics, even if they could do the problems flawlessly.

The physics education research community long ago moved to background problem solving (a little) in favor of an increased emphasis on explanation and qualitative (conceptual) understanding. I think a very under-appreciated consequence of this is greater student satisfaction with their learning, in contrast to my interviewees' high school experience. Engagement, in fact, has become a primary driver of my experimental instruction; the issue deserves a lot more work.

Mathematics education research and instruction are different cultural beasts than physics. Even if we take my anecdote's implications at face value, it's not clear the lesson is exactly the same in mathematics. However, one of mathematics education's reform agendas is moving from the paradigm of learning via constant exercise of methods and techniques, to something involving a deeper understanding of concepts, if not exactly a focus on explanation per se, which seems more evidently important to physicists. I recommend to my mathematics education colleagues yet more emphasis on this side of mathematics. 
Even methods and techniques can be generally reframed in terms of justification and invention of alternatives, rather than simply "absorbing and mastering," parallel to how I describe (in my response to Section "The Transition from Arithmetic to Algebra") an increased importance for understanding representations broadly, including student invention and judgments of aptness.

I think the continuist research program here could yield great dividends. If we can see bit by bit how various competencies co-evolve (e.g., conceptual and procedural), we will have a much more grounded understanding about how different emphases have an impact on each other. I expect, for example, that a better integration of conceptual and technical threads will alleviate apparent discontinuities, particularly in terms of the stability and perceived meaningfulness of learned procedures. "Sense-making," while occupying a strong niche in mathematics education, needs more precise definition and theoretical elaboration, which high-resolution empirical work can supply.

ONK: There is widespread agreement that the acquisition of concepts in areas such as multiplication or calculus, for example, requires both procedural and conceptual fluency (National Council of Teachers of Mathematics [NCTM], 2000). However, there is less agreement concerning the appropriate instructional balance between teaching for conceptual and procedural knowledge or how teaching can be organized to promote both types of knowledge.

I would like to mention the Inquiry-Oriented Differential Equations (IO-DE) project, which is an example of a collaborative effort between mathematics educators and mathematicians that seeks to explore the prospects and possibilities for improving undergraduate mathematics education, using differential equations as an example. Traditional differential equations courses at the university level are known as technique-driven or procedure-driven enterprises - like a cookbook. Rasmussen, Kwon, Allen, Marrongelle, and Burtch (2006) conducted an evaluation study to compare the routine skills and conceptual understandings of central ideas and analytic methods for solving differential equations between students in inquiry-oriented classes and traditionally taught classes at four undergraduate institutions in Korea and United States. Whereas IO-DE project classes at all sites typically followed an inquiry-oriented format, comparison classes at all sites typically followed a lecture-style format. The assessment consisted of procedural problems and conceptual problems. Procedural problems focused on students' instrumental understanding, such as the analytic and numerical nature of differential equations. On the other hand, conceptual understanding problems were aimed at evaluating students' understandings of important ideas and concepts. The students in the IO-DE classes scored better than the students with traditional instruction on both conceptual and procedural assessments, even though the focus of IO-DE was not on procedural and technique skills of differential equations. The more interesting data was the IO-DE students demonstrated higher retention rate on both procedural and conceptual assessments one year after the course. Our findings indicate that procedural work in the acquisition of new concepts did not lead to very good retention and also did not come with conceptual understanding of mathematical concepts (Kwon, Rasmussen, \& Allen, 2005). 
An explicit intention of IO-DE project classrooms is to create a learning environment where students routinely offer explanations of and justifications for their reasoning. As our understanding of student thinking at the transition from secondary to tertiary institutions evolves, so does our understanding of the kinds of teacher knowledge that are important for promoting student learning during transition. Beyond content knowledge, such knowledge includes awareness of students' informal and intuitive ways of reasoning about central ideas in differential equations, knowledge of pedagogical strategies that can connect to student thinking while moving the mathematical agenda forward, knowledge of theory related to social aspects of the classroom, and mathematical knowledge specific to teaching mathematics.

\section{What Is the Possible Role of the Students (or Teachers) in Helping to Ease Transitions?}

AdS: For me, the big picture here is that in the power structure of education, students are currently nearly completely disenfranchised. I feel strongly that it is imperative that we do something about it.

A former student of mine, while she was a graduate student in physics, organized a completely student-initiated and student-run program to help new undergraduates, particularly women and minorities (who are severely underrepresented in physics and other technical fields), deal with the transition from high school to university studies. Some of the best aspects of the program were that "welcoming" was a core value, and also that, freed a bit from the stodgy, self-satisfied university teaching faculty culture, they could enact instruction with a very different feel. For example, they introduced much more active, exploration-oriented instruction and talked explicitly about the epistemic nature of physics as a modeling enterprise. The program is now a national model, and there are initiatives to replicate it more widely.

I have experienced extremely positive student cultures and extremely negative ones. Positive is unambiguously better. I would like to cultivate students who are intolerant of mechanistic and disconnected instruction and who know how to - and are anxious to - engage each other in collaborative disciplinary inquiry.

I think the presumption that only teachers can "make this happen" is a symptom of endemic disrespect for students' competencies to help foster their own learning. I recognize, of course, that teachers generally have a special role in instigating and supporting student initiative. However, I advocate that we actively help develop autonomous strengths within the student community. From a scientific point of view, I don't believe that any culture we might imagine is possible to instantiate, so the "design" of classroom student cultures is not just an action to take, but a complex field we need to learn to navigate. 
LV: From my perspective, it is quite clear that the learner can help in bridging between the culture and practice of school and that of home. More particularly, learners themselves can look for activities and artifacts outside school that relate to school mathematics and vice versa. In this sense, they can act as active and constructive go-betweens to find out what mathematics is at homes and communities and document who does what kinds of mathematics in their environment.

Take, for example, Luis Moll's concept of "Funds of Knowledge," which refers to the historically accumulated and culturally developed bodies of knowledge learners can bring into the classroom because of their unique familial, cultural, and experiential backgrounds, but which also could be identified, valued, and used by teachers (González, Moll, \& Amanti, 2005), to which I referred in my talk.

Quite evidently, in this approach there should be an explicit and systematic attempt by teachers to learn more about their learners' funds of knowledge. This is primarily the responsibility of the teaching side. In the funds of knowledge approach, it is expected that teachers will try to learn more about their learners' funds of knowledge by visiting learners' home environments. It should be clear that this is absolutely not a simple task, but a task that requires a great deal of preparation and coaching.

But, of course, this kind of bridging learners' home and school environment by the teacher also requires an openness and active willingness of the learners (and their parents, particularly if we are talking about young learners, and possibly also other members of the learner's family and broader environment) to share their funds of knowledge. The literature contains several examples (cases, described in the literature) wherein this has been successfully realized (building houses, making candy and so on; e.g., Sandova-Taylor, 2005).

In addition, as critical math educators such as Gutstein, Greer, and Mukhopadhyay have argued, such bridging activities, if properly handled by the teacher, may not only help to establish the relevance of mathematics but also create useful stepping stones for mathematical knowledge building as such. It may also help to demonstrate and validate its non-elitist existence and to create respect for multiple forms of mathematical practices. Thinking of Freire's quote that "Intellectual activity of those without power is always characterized as non-intellectual," one can replace "intellectual" with "mathematical" to readily see that "Mathematical activity of those without power is always characterized as non-mathematical" (Greer and Mukhopadhyay, personal communication). Therefore, establishing such bridging activities may reinforce in learners the belief that mathematics is a universal human activity and is done actively by all kinds of people. Furthermore, when learning more about learners' familial, cultural, and experiential backgrounds and trying to link these backgrounds to school mathematics, themes such as AIDS, poverty, sexual exploitation, and pollution, ... may pop up, and learners may start to realize how mathematics can become a helpful, even critical, element in their process of emancipation or conscientization (again in the Freirean sense). 
ONK: I think that the role of teachers at both institutions is more important than the students' role in helping ease transitions. The essence of mathematical knowledge cannot and should not be compartmentalized into school mathematics and university mathematics. School mathematics is a subset of university mathematics. Teachers should facilitate the interconnection between secondary and tertiary schooling on specific topics in calculus, linear algebra, and analysis. University teachers need to view elementary mathematics from a higher standpoint. In addition, students should appreciate the need for a different kind of mathematics at the university level. They should at the same time understand how this new mathematics is related to school mathematics, why it is different, and why it nevertheless has potential in contributing to the development of students' mathematical competence in a way that makes it useful for qualified mathematics teaching at the school level.

Furthermore, Kwon, Rasmussen, Marrongelle, Park, Cho, and Park (2008) focused on teacher revoicing because it is one of the discursive strategies that often occurs in the teaching of mathematics but which has received limited attention in mathematics education research at the undergraduate level. Our analysis shows that teacher revoicing can constitute a major part of teachers' repertoires of discursive moves and carries out critical functions in the context of mathematics practice in class. From this perspective, revoicing can serve at least three functions in the classroom. First, revoicing functions to highlight specific mathematical ideas and/or provide mathematical content to move the mathematical agenda forward. Second, revoicing functions to honor and empower student thinking. That is, revoicing facilitates the development of students' mathematical identities. Third, revoicing functions to help students understand what constitutes a sufficient explanation or justification. That is, revoicing can serve to promote certain social and socio-mathematical norms. In their learning environment, students learn new mathematics by inquiry, which involves solving novel problems, debating mathematical solutions, posing and following up on conjectures, and explaining and justifying one's thinking.

\section{Conclusion}

While the presentations during the first part of the panel showed that the different perspectives tended to address different questions, the answers presented here to identical questions show consequences of the choice of perspectives in terms of differences in the answers.

Most of the time, the answers of the panelists complemented each other. Whatever approach was chosen, there was a consensus acknowledging the complexity of transition phenomena. The transition is not composed of an initial state, a final state, and a gap in between that can be spanned by an appropriate bridge. Instead, it is a complex, cumulative path to be managed. 
A micro-level must be taken into account, separating different dimensions in the learning and teaching processes, which is a necessary first step to understanding the transition process and to analyze it as an incremental path. At the same time, separating different dimensions does not mean considering them independently: For example, the procedural and conceptual aspects are certainly two dimensions in the learning of mathematics, but they are strongly linked and their interactions within transition processes constitute an important issue. A macro-level must also be considered, for example, to analyze how curriculum choices at a large scale, encompassing several institutions, shape the transition.

Moreover the "initial" and "final" states must not be seen as two clearly separate points but as two zones whose frontiers are more or less clear and which can have intersections. These intersections are linked by the existence of boundary objectsobjects present in both zones - and/or of brokers-persons living in both zones. The different approaches focus on different kinds of boundary objects or brokers: mathematical problems existing in and out of school and common work between teachers of different institutions trying to understand each other's mathematical practices and to bring them closer. Nevertheless, all the approaches suggest that trying to identify or to develop boundary objects is a promising direction for research, especially with an aim of proposing solutions to ease the transitions' difficulties.

Sometimes the different approaches also lead to (apparently) conflicting answers. Teachers are certainly central actors shaping the teaching and learning processes, thus also in shaping the transition processes. A focus on teachers alone, though, can suggest that students cannot themselves act on transition processes, but examples exist showing the possible actions of students. Moreover, teachers can work with students to ease the transition — or, with a different perspective (Sensevy, Gruson, \& Forest, 2015), the joint action of teachers and students can ease some transition processes.

This discussion shows, in any case, that mathematical research on transition is lively and rich and that further research directions are open. We hope that this panel will serve as a resource for those who want to pursue these directions!

\section{References}

Bosch, M. (2015). Doing research within the anthropological theory of the didactic: the case of school algebra. In S. J. Cho (Ed.), Selected regular lectures from the 12th international congress on mathematical education (pp. 51-69). New York: Springer.

Cognition and Technology Group at Vanderbilt. (1997). The Jasper project: Lessons in curriculum, instruction, assessment, and professional development. Mahwah, NJ: Erlbaum. 
Crafter, S., \& Maunder, R. (2012). Understanding transitions using a sociocultural framework. Educational and Child Psychology, 29(1), 10-18.

diSessa, A. A. (2004). Meta-representation: Native competence and targets for instruction. Cognition and Instruction, 22(3), 293-331.

Dorier, J.-L. (Ed.). (2000). On the Teaching of Linear Algebra. Dordrecht: Kluwer Academic Publishers.

Fonseca, C., Bosch, M., \& Gascón, J. (2004). Incompletitud de las organizaciones matemáticas locales en las instituciones escolares. Recherches en didactique des mathématiques, 24(2), 205-250.

González, N., Moll, L. C., \& Amanti, C. (Eds.). (2005). Funds of knowledge: Theorizing practices in households, communities, and classrooms. Mahwah, NJ: Erlbaum.

Gravemeijer, K. (1997). Solving word problems: A case of modelling? Learning and Instruction, 7, 389-397.

Gueudet, G., Bosch, M., diSessa, A., Kwon, O.-N., \& Verschaffel, L. (2016). Transitions in mathematics education. Berlin: Springer.

Hacker, A. (2012). Is algebra necessary? The New York Times (Sunday review).

Kwon, O. N., Rasmussen, C., \& Allen, K. (2005). Students' retention of mathematical knowledge and skills in differential equations. School Science and Mathematics, 105(5), 1-13.

Kwon, O. N., Ju, M. K., Rasmussen, C., Marrongelle, K., Park, J. H., Cho, K. Y., et al. (2008). Utilization of revoicing based on learners' thinking in an inquiry-oriented differential equations class. The SNU Journal of Education Research, 17, 111-134.

Levin (Campbell), M. E. (2012). Modeling the co-development of strategic and conceptual knowledge during mathematical problem solving. (Unpublished doctoral dissertation). Berkeley: University of California.

National Council of Teachers of Mathematics. (2000). Principles and standards for school mathematics. Reston VA: Author.

Paulos, J. A. (2001). Innumeracy: Mathematical illiteracy and its consequences. New York, NY: Hill and Wang.

Rasmussen, C., Kwon, O. N., Allen, K., Marrongelle, K., \& Burtch, M. (2006). Capitalizing on advances in mathematics and K-12 mathematics education in undergraduate mathematics: An inquiry-oriented approach differential equations. Asia Pacific Education Review, 7(1), 85-93.

Ríordáin, M. N., \& O’Donoghue, J. (2011). Tackling the transition-the English mathematics register and students learning through the medium of Irish. Mathematics Education Research Journal, 23(1), 43-65.

Sandova-Taylor, P. (2005). Home is where the heart is. Planning a Funds of Knowledge based curriculum module. In T. González, L. C. Moll, \& C. Amanti (Eds.), Funds of Knowledge: Theorizing practices in households, communities, and classrooms (pp. 153-165). Mahwah, NJ: Erlbaum.

Sensevy, G., Gruson, B., \& Forest, D. (2015). On the nature of the semiotic structure of the didactic action: The joint action theory in didactics within a comparative approach. Interchange, 46(4), 387-412.

Serrano, L., Bosch, M., \& Gascón, J. (2007). Diseño de organizaciones didácticas para la articulación del bachillerato con el primer curso universitario. In L. Ruiz-Higueras, A. Estepa, \& F. J. García (Eds.), Sociedad, Escuela y Matemáticas. Aportaciones de la Teoría Antropológica de lo Didáctico (pp. 757-764). Jaén: Servicio de Publicaciones de la Universidad de Jaén.

Sierpinska, A. (2006). Sources of students' frustration in bridging mathematics courses. In Proceedings of 30th Conference of the International Group for the Psychology of Mathematics Education (Vol. 5, pp. 121-129). 
Tall, D. (2002). Continuities and discontinuities in long-term learning schemas. In Tall, D. \& Thomas, M. (Eds.) Intelligence, learning and understanding: A tribute to Richard Skemp (pp. 151-177). Flaxton QLD, Australia: Post Pressed.

Verschaffel, L., Greer, B., \& De Corte, E. (2000). Making sense of word problems. Lisse, The Netherlands: Swets \& Zeitlinger.

Open Access Except where otherwise noted, this chapter is licensed under a Creative Commons Attribution 4.0 International License. To view a copy of this license, visit http://creativecommons. org/licenses/by/4.0/. 